\title{
Experimental and numerical investigation of aerodynamics of a pneumatic nozzle for suspension fuel
}

\author{
S.V. Alekseenko ${ }^{1}$, I.S. Anufriev ${ }^{1,3}$, A.A. Dekterev ${ }^{1,2}$, V.A. Kuznetsov ${ }^{1,2}$, L.I. Maltsev ${ }^{1}$, \\ A.V. Minakov ${ }^{1,2}$, M.Yu. Chernetskiy ${ }^{1}$, E.Yu. Shadrin ${ }^{1}$, and O.V. Sharypov ${ }^{1}$ \\ ${ }^{1}$ Kutateladeze Institute of Thermophysics SB RAS, Novosibirsk, Russia \\ ${ }^{2}$ Siberian Federal University, Krasnoyarsk, Russia \\ ${ }^{3}$ Novosibirsk State Technical University, Novosibirsk, Russia
}

One of the topical problems of coal power engineering both from the point of increasing efficiency and ecological safety of heat energy production and need to utilize low-grade coals and coal wastes is the development of combustion technology for coal in the form of a coal-water suspension (CWS). The technologies of CWS combustion place high demands to the spraying devices (nozzles): absence of narrow fuel channels, which can be locked; low fuel velocities relative to the solid walls, which will reduce abrasive wear. The authors of the paper propose a pneumatic nozzle based on the use of the properties of near-wall and cumulative jets of liquid and gas and Coanda effect, which meets the basic requirements for CWS injectors. Aerodynamics control plays the determining role in the efficiency of pneumatic nozzle operation. In this paper, the structure of a single-phase gas flow in the proposed pneumatic nozzle is studied under different regime parameters using the experimental and numerical methods. The studies were carried out using the particle image velocimetry (PIV) and mathematical modeling of the flows by means of the DES and RSM turbulence models. It is shown that in the entire investigated range of excess air pressure, the converging annular jet turns into the concentrated one and forms the direct and return cumulative axial jets in the nozzle near the axis of symmetry. Due to interaction of the return and annular jets in the diffuser, a toroidal vortex is formed. At operation of a liquid fuel nozzle, such a return flow will contribute to the effective destruction of a liquid jet and formation of a highly dispersed two-phase flow. With an excess air pressure in the nozzle of 1 bar, a sound annular converging jet is formed at the nozzle outlet; with a further increase in pressure, the outflowing jet becomes supersonic, the oblique shock waves are formed there, and the axial jet acquires a barrel-like shape with formation of the Mach disks. Such a complex spatial arrangement of the flow (both in the toroidal vortex and outside it) ensures efficient dispersion of liquid fuel in this nozzle.

Key words: nozzle for coal-water suspension, dispersion of liquid, flow structure, velocity measurements, particle image velocimetry, numerical simulation, DES, RSM. 


\section{Introduction}

By now, almost all coal produced in the world is sorted and enriched; as a result, huge volumes of low-grade coals and waste of coal cleaning accumulate. Coal wastes either accumulate, polluting the environment, or they are used as raw coal in heat and power engineering, if high-grade coal is added. At the same time, they burn badly and boilers working on these coals have low efficiency. The technology of coal combustion in the form of coal-water suspension (CWS-technology) allows coal waste utilization, increasing the burning efficiency of low-grade coals [1-3]. Along with this, alternative suspension fuels, consisting of water, crushed coal (or combustible waste of its processing), chemical additives, and refinery waste are actively used now to produce energy $[4,5]$. Such a fuel is called an organic coal-water (OCWF) or composite liquid fuel (CLF). At present, a high level of solving the technical problems in preparing CWS compositions, whose characteristics allow their application at power facilities, has been achieved [6-10].

The main way to combust slurry fuels is their pre-spraying in the combustion chamber. Spraying increases significantly the contact area of fuel with oxidant and intensifies heat and mass transfer processes. A large number of nozzles, using hydraulic, mechanical, pneumatic, acoustic, electrostatic, ultrasonic, pulsating and other principles of liquid dispersion of liquids have been developed in the world. As a rule, pneumatic nozzles are used for CWS spraying because of their relative constructive and operational simplicity and reliability.

In practical applications, the nozzles must ensure efficient dispersion of fuel, given shape of the flame, and certain range of variation in fuel consumption. The presence of a fine-grained solid phase in the CWS leads to significant erosion of nozzle elements; therefore, the increased requirements are imposed on the construction of nozzles for CWS. First of all, there should be no narrow channels in the CWS sprayers, in order to avoid their locking and high velocity of suspension flow near the walls.

The group of researchers at the Institute of Thermophysics of the Russian Academy of Sciences has proposed a pneumatic nozzle $[11,12]$ based on using the properties of wall and cumulative jets and the Coanda effect. Its design meets the requirements for devices of CWS spraying. Schematically, this nozzle is shown in Fig. 1. A gas jet from a conical slit nozzle 6 forms a converging jet gas flow outside the nozzle. As a result of conical jet convergence, the flow along the nozzle axis is generated in the forward direction, and a high-speed return jet of the cumulative type is formed. The supply of liquid fuel under the pressure through central pipe 3 leads to formation of a liquid jet flowing out of the neck of diffuser nozzle 4 . Due to the Coanda effect, the liquid flow deviates to the walls of diffuser nozzle 4 and spreads along them as a thin layer. Disruption of liquid jet integrity is facilitated by a high-speed return jet of gas. Together with the Coanda effect, this leads to uniform distribution of liquid along the diffuser walls in the form of a thin film. At that, the return gas jet after collision with liquid changes its direction again to the opposite one, spreads along the diffuser walls and accelerates the liquid flow. Inside the cone, a toroidal vortex is formed in the gas flow. Due to interaction of gas and liquid flows, the fine-dispersed gas-droplet flow is formed at the 
diffuser outlet. Some small droplets of liquid enter the return gas jet. At that, the average density of return flow increases as compared to pure gas, and efficiency of destruction of the concentrated liquid jet increases.

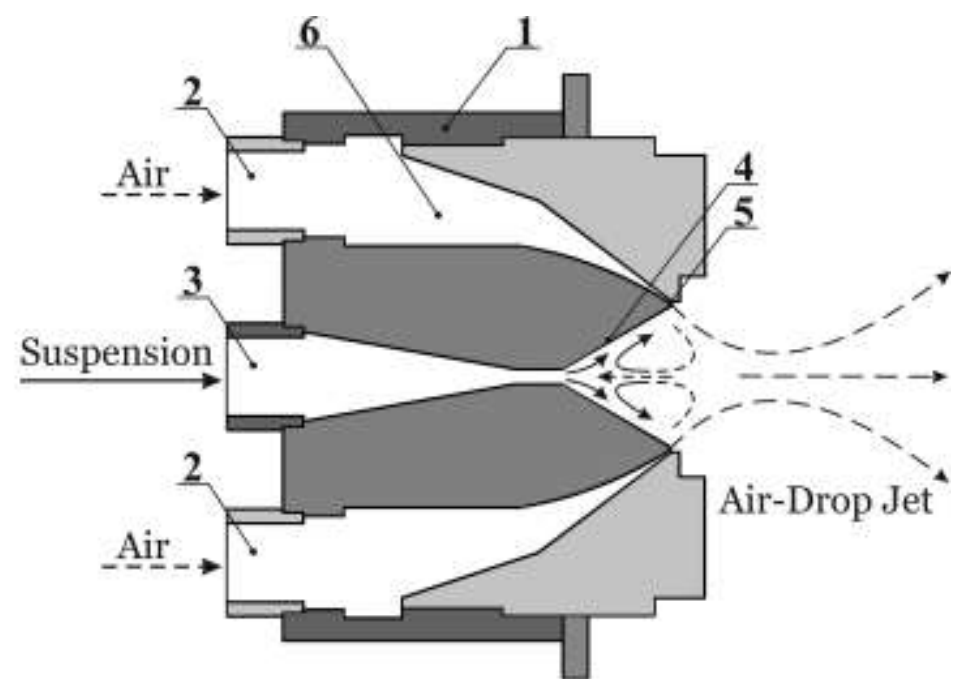

Fig. 1. Scheme of pneumatic nozzle: case (1); tube for gas supply (2); tube for liquid (coal-water suspension) supply (3); diffusion nozzle (4); annular gas nozzle (width of $0.8 \mathrm{~mm}$, diameter of $28 \mathrm{~mm}$ ) (5); gas chamber (6).

It is obvious that the efficiency of this nozzle operation is determined by organization of aerodynamics. To substantiate the ways of the process control, obtain the dependencies of the flame divergence angle and disperse composition of fuel on the design characteristics of the nozzle and regime parameters, the experimental and computational studies are required. Some principal questions of nozzle operation are considered in [12]. Although this nozzle is used at industrial power facilities [1], until now there were no systematic studies of hydro-gas-dynamic processes ensuring nozzle operation, required for optimization of operating conditions. In this paper, as a first step, the structure of the gas flow, leaving the pneumatic nozzle of the proposed design, is studied experimentally and theoretically under various regime parameters without liquid (suspension) supply. 


\section{Experimental setup and measurement methods}

Experimental studies were carried out at a setup, whose scheme is shown in Fig. 2-a. The flow velocity field was measured by the particle image velocimety (PIV) method using the 2D PIV system "Polis" (Fig. 2-b) [13]. This measuring complex includes: double pulse Nd: YAG laser QuantelEVG (pulse energy of $145 \mathrm{~mJ}$, maximal pulse frequency of $15 \mathrm{~Hz}$, pulse duration of $10 \mathrm{~ns}$ ); lens for laser sheet forming; CCD camera ImperX B4820-M (resolution of 4904x3280 pixels, shooting frequency of $3.2 \mathrm{~Hz}$, minimal interframe delay of $200 \mathrm{~ns}$ ); macro lens Sigma AF 180mm f/2.8 Macro; synchronizing processor; personal computer with ActualFlow software and PIV Kit; and mounting kit based on Newport elements.

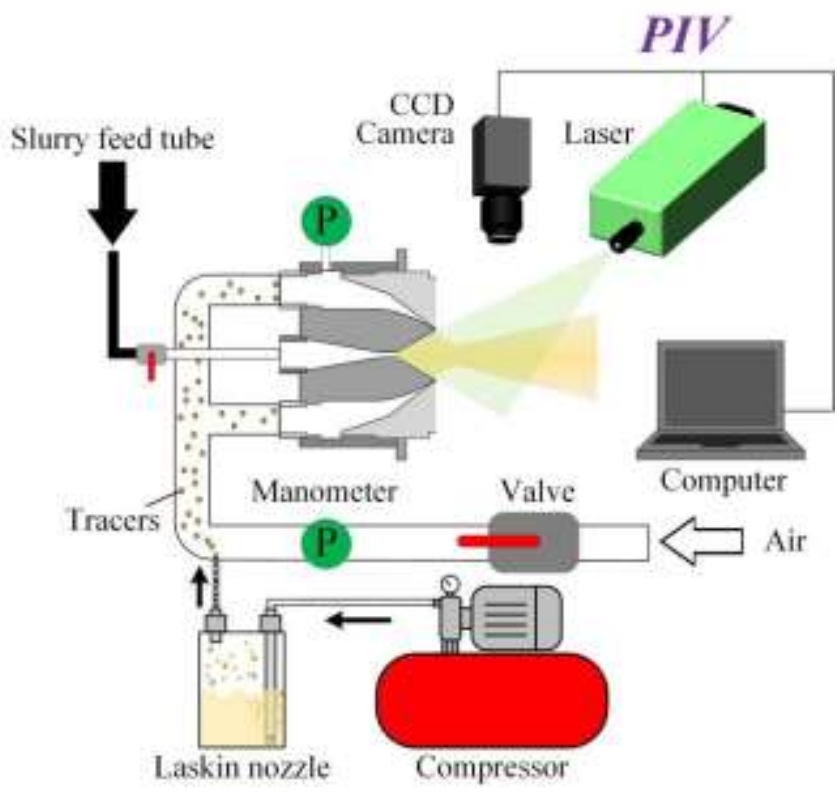

(a)

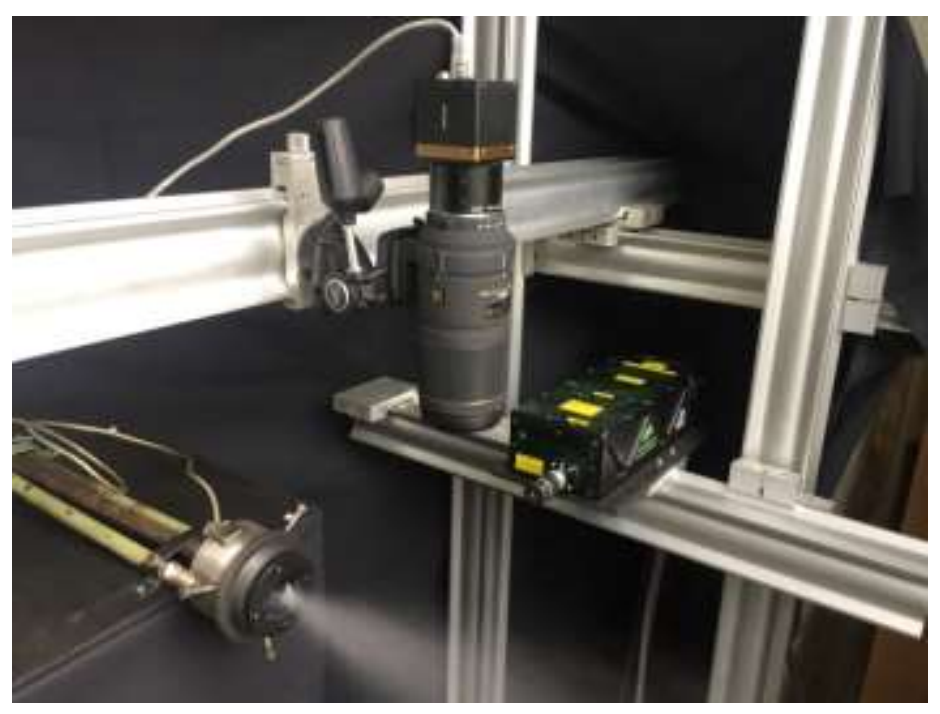

(b)

Fig. 2. (a) Scheme of experimental setup; (b) experimental setup with the PIV-system "POLIS".

In this paper, we investigate the structure of a single-phase gas flow. In PIV-measurements, the compressed air flows into the atmosphere through the annular nozzle orifice, the liquid supply tube is blocked. To seed the tracers into the flow, we used microscopic drops of vegetable oil, created through the Laskin nozzle, and fed into the main air flow (Fig. 2-a).

In experiments, we studied the regimes with the initial air pressure in the annular gas chamber of the nozzle in the range of 0.5-3.0 bar. The pressure was regulated by means of a valve cock and controlled by a standard manometer (Fig. 2-a). We chose this pressure range because at initial air pressure below 0.5 bars, strong pulsations of jet are observed and the flow is unstable. At the pressure above $3.0 \mathrm{~atm}$. the flow velocity at the outlet of the annular nozzle is higher than $470 \mathrm{~m} / \mathrm{s}$, and this requires the use of special recording equipment [14] with an interframe delay of less than 200 ns. The selected range of the carrier phase flow corresponds to nozzle operation on the real boiler. 
The velocity field was measured in a horizontal cross-section passing through the axis of symmetry of the nozzle. A series of 1000 pairs of frames with the frequency of $2 \mathrm{~Hz}$, time of the first frame exposure of $100 \mu \mathrm{s}$, and delay time between the first and second frames in a pair of 1-2 $\mu$ s (depending on the regime) were obtained for each regime. An iterative cross-correlation algorithm for calculating the velocity fields was used to process the data, dividing the computational domain into 64x64-pixel cells with a spatial overlap of 50\%. To suppress background illumination, the algorithm of digital image filtering ("background subtraction") was used. The spatial grid pitch was about 1x1 mm.

\section{Mathematical model and numerical method}

A turbulent supersonic flow of a compressible gas is considered here. To simulate turbulence, the Reynolds stress model (RSM) was used as the main model $[15,16]$. In general, the equations of this model have the form:

$$
\begin{gathered}
\frac{\partial u_{i}}{\partial x_{i}}=0 \\
\frac{\partial\left(\rho u_{i}\right)}{\partial t}+\frac{\partial \rho u_{i} u_{j}}{\partial x_{j}}=-\frac{\partial p}{\partial x_{i}}+\frac{\partial}{\partial x_{j}}\left(\mu\left(\frac{\partial u_{i}}{\partial x_{j}}+\frac{\partial u_{j}}{\partial x_{i}}\right)-\rho \overline{u_{i}^{\prime} u_{j}^{\prime}}\right), i=1,2,3
\end{gathered}
$$

In the RSM model, the components of $\overline{u_{i}^{\prime} u_{j}^{\prime}}$ tensor of Reynolds stresses are determined from the solution to transfer equations:

$$
\frac{\partial}{\partial t}\left(\rho \overline{u_{i}^{\prime} u_{j}^{\prime}}\right)+\frac{\partial}{\partial x_{l}}\left(\rho u_{l} \overline{u_{i}^{\prime} u_{j}^{\prime}}\right)=\rho\left(P_{i j}+D_{i f f}-\varepsilon_{i j}+\phi_{i j}\right) .
$$

In the right part of equation, there are the terms responsible for generation $\left(P_{i j}\right)$, diffusion ( $\left.\operatorname{Diff}_{i j}\right)$, dissipation $\left(\varepsilon_{i j}\right)$, and redistribution $\phi_{i j}$. Exact expressions for these terms contain triple correlations of velocity pulsations; therefore, some hypotheses are adopted to close the system of equations for the terms in the right part. The exception is the generation tensor, which does not require modeling and is determined as follows:

$$
P_{i j}=-\left(\overline{u_{i}^{\prime} u_{l}^{\prime}} \frac{\partial u_{j}}{\partial x_{l}}+\overline{u_{j}^{\prime} u_{l}^{\prime}} \frac{\partial u_{i}}{\partial x_{l}}\right)
$$

At a distance from the wall, dissipation tensor is taken the isotropic one:

$$
\varepsilon_{i j}=\frac{2}{3} \delta_{i j} \varepsilon,
$$

where $\varepsilon$ is the rate of dissipation of the kinetic energy of turbulent pulsations. This value is determined from the transfer equation, corresponding to the similar equation in turbulence model $k-\varepsilon$ : 


$$
\begin{gathered}
\frac{\partial \rho \varepsilon}{\partial t}+\frac{\partial p}{\partial x_{j}}\left(\rho u_{j} \varepsilon\right)=\frac{\partial}{\partial x_{j}}\left(\mu \frac{\partial \varepsilon}{\partial x_{j}}+C_{\varepsilon} \rho T \overline{u_{i}^{\prime} u_{j}^{\prime}} \cdot \frac{\partial \varepsilon}{\partial x_{i}}\right)+\rho \frac{C_{\varepsilon 1} P_{k}-C_{\varepsilon 2} \varepsilon}{T}, \\
T=\frac{k}{\varepsilon}, C_{\varepsilon}=0.183, C_{\varepsilon 1}=1.44, C_{\varepsilon 2}=1.83
\end{gathered}
$$

Here, $T$ is integral time scale of turbulence. This equation includes kinetic energy of turbulence $k$ and rate of its generation $P_{k}$. They are found as the sum of diagonal components of the corresponding tensors:

$$
P_{k}=\frac{1}{2} P_{i i}, k=\frac{1}{2} \overline{u_{i}^{\prime} u_{i}^{\prime}} .
$$

The diffusion term is modeled by generalized gradient hypothesis [17]:

$$
\operatorname{Diff}_{i j}=\frac{\partial}{\partial x_{k}}\left(\mu \frac{\partial \overline{u_{i}^{\prime} u_{j}^{\prime}}}{\partial x_{k}}+C_{s} \rho T \overline{T \overline{u_{k}^{\prime} u_{l}^{\prime}}} \frac{\partial \overline{u_{i}^{\prime} u_{j}^{\prime}}}{\partial x_{l}}\right) .
$$

The central element of the Reynolds stress model is the term of redistribution responsible for the transition of some tensor components to others. In this paper, the SSG model is used to describe it [18]. The redistribution term is the sum of the "slow" and "fast" parts, which depend quadratically on the strain rate tensor and anisotropy tensor. The first part looks like:

$$
\phi_{i j, 1}=-c_{1} \varepsilon\left\{a_{i j}-c_{1}^{\prime}\left(a_{i l} a_{l j}-\frac{1}{3} A_{2} \delta_{i j}\right)\right\},
$$

and second part is:

$$
\phi_{i j, 2}=-c_{2}\left(P_{i j}-\frac{2}{3} P_{k} \delta_{i j}\right)-c_{3}\left(D_{i j}-\frac{2}{3} D_{k} \delta_{i j}\right)-c_{4} k S_{i j}-2 c_{5} a_{i j} P_{k},
$$

where

$$
D_{i j}=-\left(\overline{u_{i}^{\prime} u_{l}^{\prime}} \frac{\partial u_{l}}{\partial x_{j}}+\overline{u_{j}^{\prime} u_{l}^{\prime}} \frac{\partial u_{l}}{\partial x_{i}}\right), D_{k}=\frac{1}{2} D_{i i}
$$

anisotropy tensor:

$$
a_{i j}=\frac{\overline{u_{i}^{\prime} u_{j}^{\prime}}}{k}-\frac{2}{3} \delta_{i j},
$$

second invariant of anisotropy tensor:

$$
A_{2}=a_{i j} a_{i j}
$$

closing constants:

$$
c_{1}=1.7, c_{1}^{\prime}=0.617, c_{2}=0.4125, c_{3}=0.2125, c_{4}=0.033+0.65 \sqrt{A_{2}}, c_{5}=0.45 .
$$


In addition to the motion equations, the mathematical model includes the equation of total energy balance, written as:

$$
\frac{\partial}{\partial t}(\rho E)+\nabla \cdot(\vec{v}(\rho E+p))=\nabla \cdot\left(k_{e f f} \nabla T\right)
$$

where $k_{\text {eff }}$ is effective coefficient of heat conductivity.

Expression for total energy takes form:

$$
E=h-\frac{p}{\rho}+\frac{v^{2}}{2}
$$

where $h$ is enthalpy. For the ideal gas:

$$
h=\int_{T_{r e f}}^{T} c_{p, j} d T
$$

where $T_{r e f}=298.15 \mathrm{~K}$.

In addition to the RSM RANS model of turbulence, a vortex-resolving method (DES) was considered. The DES method combines the well-known approaches of RANS and LES. In flow areas, where the cell size of the computational grid $\Delta$ is sufficient to resolve energy-carrying vortices, i.e., at $\Delta<L_{t}$, the LES method is used, and in the rest area, we apply RANS. The RANS models work in the boundary layers, and in the rest area, it is LES. In this paper, the DES method [19], based on the $k-\omega$ SST Menter model [20], is used. In this model, the dissipative term in the equation of kinetic energy of turbulent pulsations is limited by the $F_{\text {DES }}$ switch:

$$
\begin{gathered}
\frac{\partial(\rho k)}{\partial t}+\nabla \cdot(\rho \mathbf{v} k)=P_{k}-\beta^{*} \rho k \omega \cdot F_{D E S}+\nabla \cdot\left(\mu_{e f} \nabla k\right) \\
F_{D E S}=\max \left(L_{t} /\left(C_{D E S} \Delta\right), 1\right), l_{t}=\frac{k^{1 / 2}}{\beta^{*} \omega}, C_{D E S}=0.61
\end{gathered}
$$

The previous calculations show that this method makes it possible to describe reliably large-scale turbulent pulsations for various jet flows for an acceptable calculation time [21-23].

The equations of state of an ideal gas were used in calculations. At that, the thermophysical properties of air were set taking into account the temperature dependence. In particular, heat capacities of gases were determined according to the polynomial dependence; the molecular viscosity was determined by the Sutherland dependence.

The numerical algorithm is based on the finite volume method for a grid with the cells of an arbitrary shape. The convective terms of the transport equations for the RSM model are approximated using a counter-current scheme of the second order; for the DES model, a limited central-difference scheme is used. Approximation of diffusion flows and source terms has the second order of accuracy. The non-stationary components in DES were approximated by an implicit scheme of the second order of accuracy. The time step was chosen from CFL $<2$ condition. The relationship between the velocity and pressure fields was 
implemented using the SIMPLEC procedure on the combined grids. To eliminate oscillations of the pressure field, the Phy-Chow approach is used, which involves special interpolation of the velocity vector on the faces of control volumes. The resulting system of difference equations was solved by an iterative method using a multigrid solver.

\section{Problem statement for numerical simulation}

For the RSM model, the problem was solved in axisymmetric stationary statement. The geometry of computational domain is shown in Figure 3-a. For the DES model, calculation was carried out in the 3D statement. The geometry of computational domain in this case was obtained by rotating the axisymmetric region around its axis (see Fig. 3b). The width of the annular gap was $0.8 \mathrm{~mm}$. The output conditions with a fixed pressure were set at all outer boundaries of computational domain as the boundary conditions. The conditions of a solid wall were set on the outer and inner surfaces of the nozzle. At the inlet to the nozzle (tubes for gas injection and liquid supply), a fixed value of the excess gas pressure was set. In this work, the excess gas pressure was varied from 1 to 5 bars.

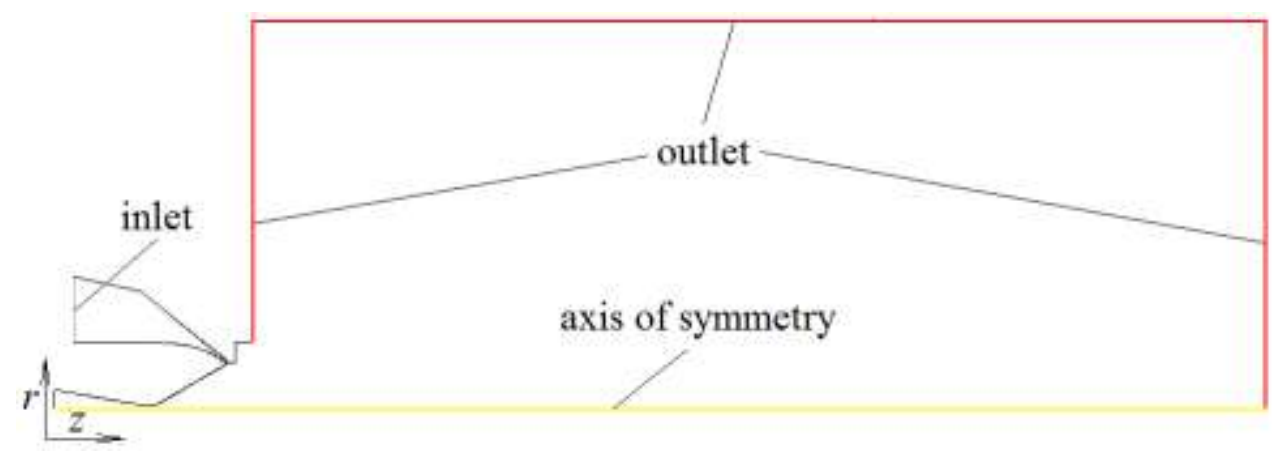

a)

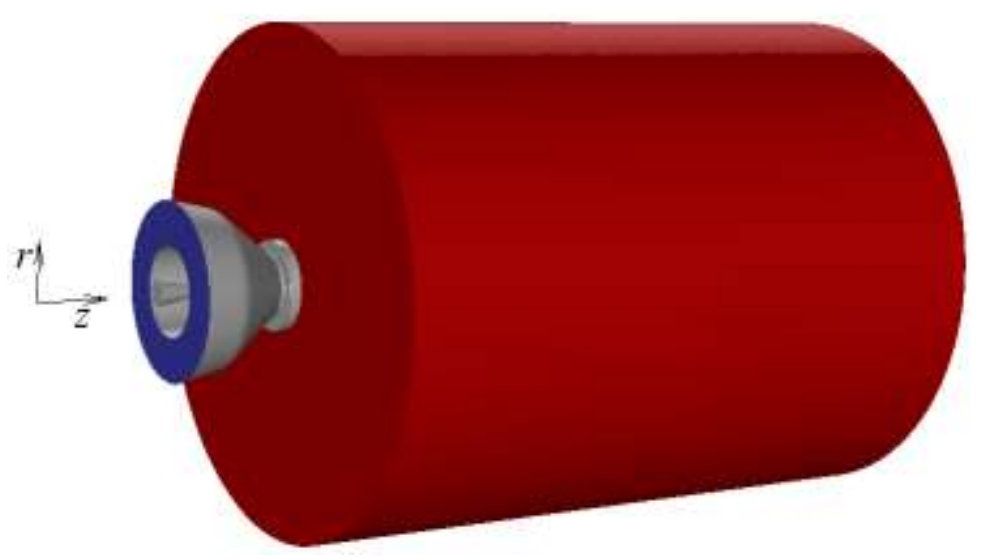

b)

Fig. 3. Geometry of computational domain for the RSM model (a) and DES model (b).

For the calculation, we used the structured computational grids with local refining near the diffuser nozzle and region of jet formation. The computational grid in the axisymmetric case is shown in Fig. 4. 
General detailing of this computational grid in the axisymmetric case was 200000 knots. As it can be seen, this grid is strongly condensed in the region of the jet. To solve the shock-wave cells formed in the jet, the technology of gradient adaptation of the computational grid was applied for RSM. With the help of this technology, the computational grid in the calculation process is automatically condensed in the region of high gradients of solution. In this case, the density gradient was chosen as the controlling parameter. An example of how the original grid changes during gradient adaptation is shown in Fig. 5 for an excess pressure of 3 bars. Cells in the region of high gradients are four times smaller than in the original grid. As a result, the total number of design nodes in the optimized grid is close to 800,000 cells. The use of such a detailed grid allowed solving the oblique shock waves and shock-wave cells in the considered nozzle. In the three-dimensional case, the computational grid for the DES method contained about 8 million cells. In the cross-section, the structure of the computational grid is similar to the grid shown in Fig. 4, but more sparse. Gradient adaptation of the grid for the DES method was not applied, the grid was fixed.

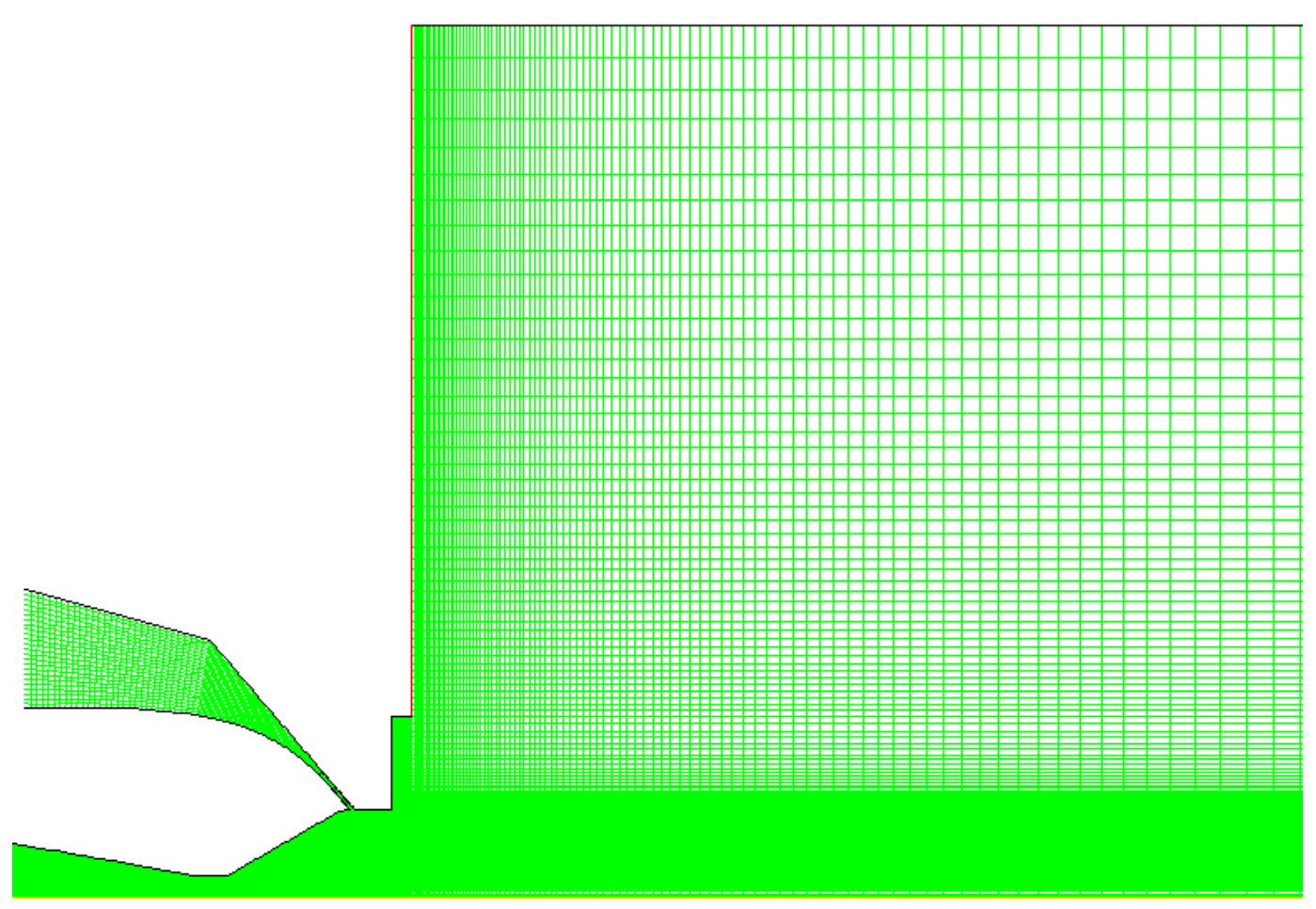

Fig. 4. Computational grid. 


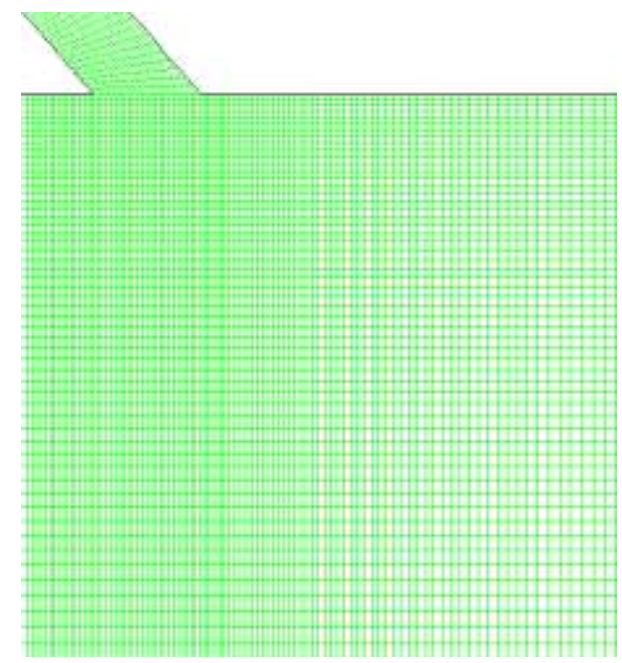

a)

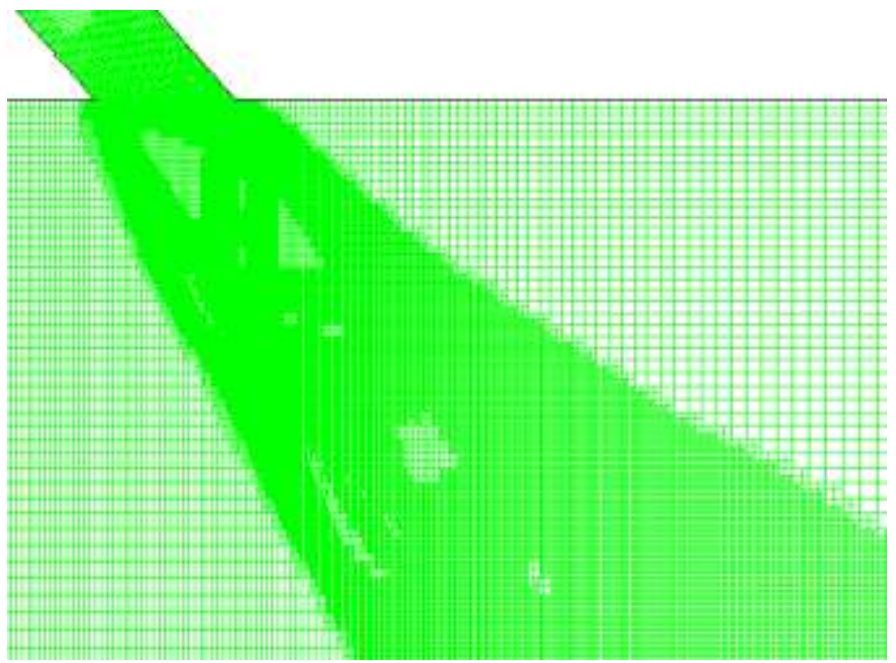

b)

Fig. 5. Computational grid at the nozzle outlet for the RSM model:

a) initial grid, b) with gradient adaptation.

\section{Investigation results and analysis}

When visualizing the flow, water was fed through the tube for liquid supply (see Fig. 1). A characteristic view of the gas-droplet jet formed during liquid dispersion in the studied pneumatic nozzle in shown in Fig. 6 for various regimes. The images shown in Fig. 6 were obtained in experiments with the water flow rate of $200 \mathrm{~kg} / \mathrm{h}$. The results demonstrate that in the excess pressure range of 1-3 bars, the characteristic opening angle of the jet is $20-25^{\circ}$ (Fig. 6). The next results of PIV-measurements and numerical calculations, as was noted above, were obtained for a single-phase gas flow (no liquid was fed).

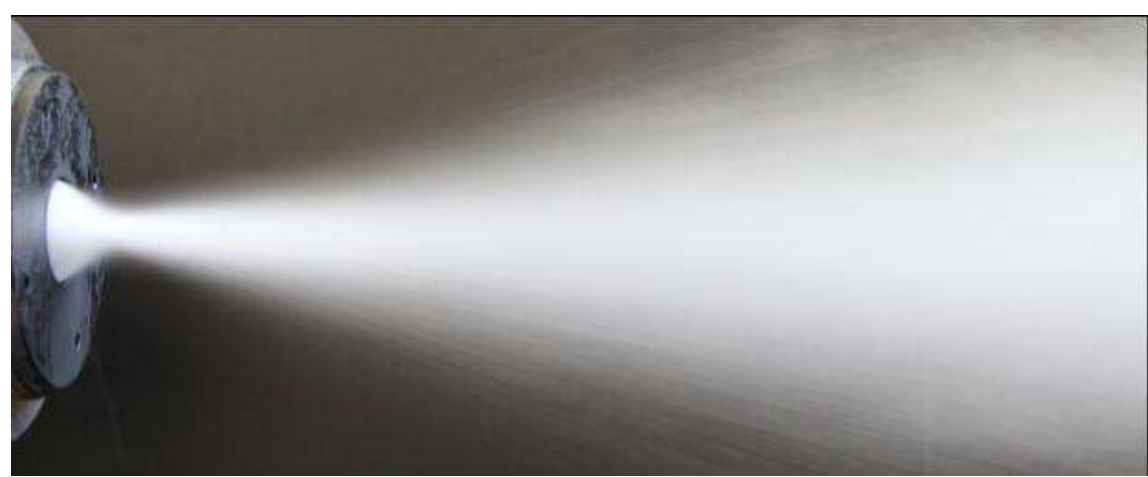

(a)

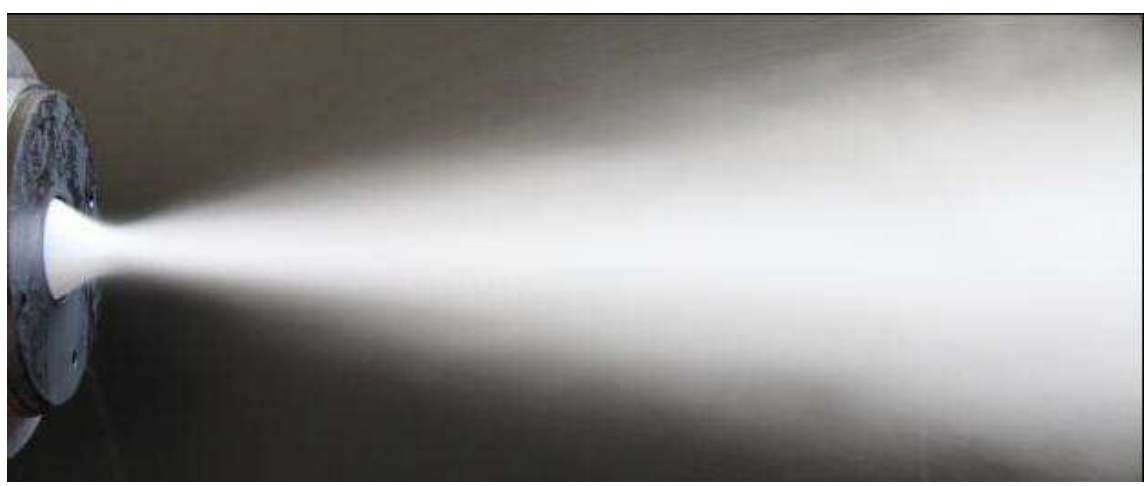

(b) 


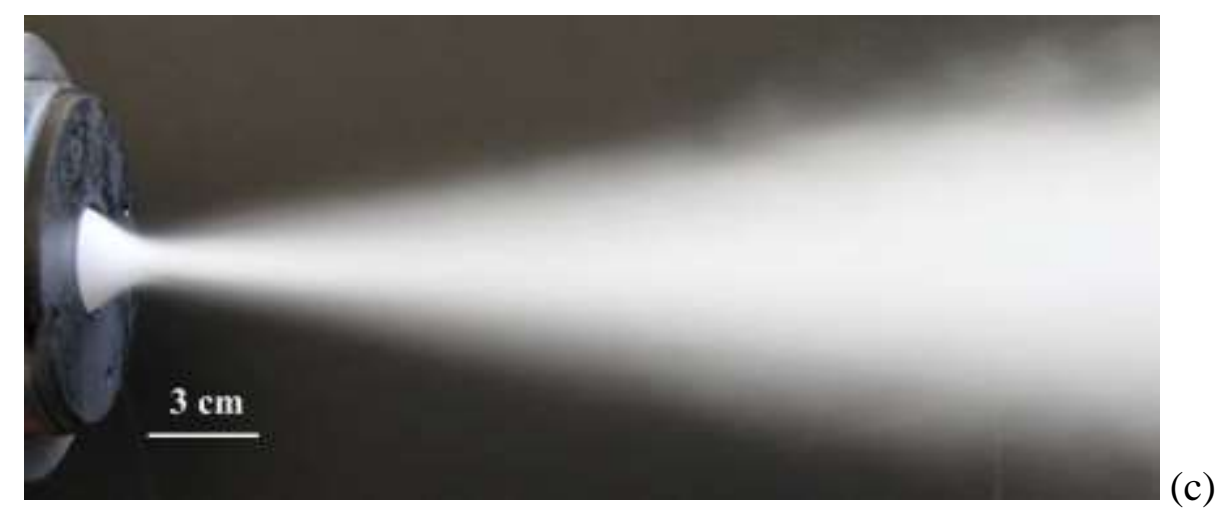

Fig. 6. Visualization of the gas-droplet flow for different values of excess pressure: (a) $P_{0}=1$ bar; (b)

$$
P_{0}=2 \text { bar; (c) } P_{0}=3 \text { bar. }
$$

As it is known, the gas dynamic structure of supersonic gas jets depends on the calculated Mach number at the nozzle edge (M), geometric profile of supersonic part of the nozzle, and the ratio of pressures in the nozzle pre-chamber and surrounding space. Investigation results on the air flows from the considered nozzle are presented in Figs. 7-15 for different values of excess pressure in the nozzle.

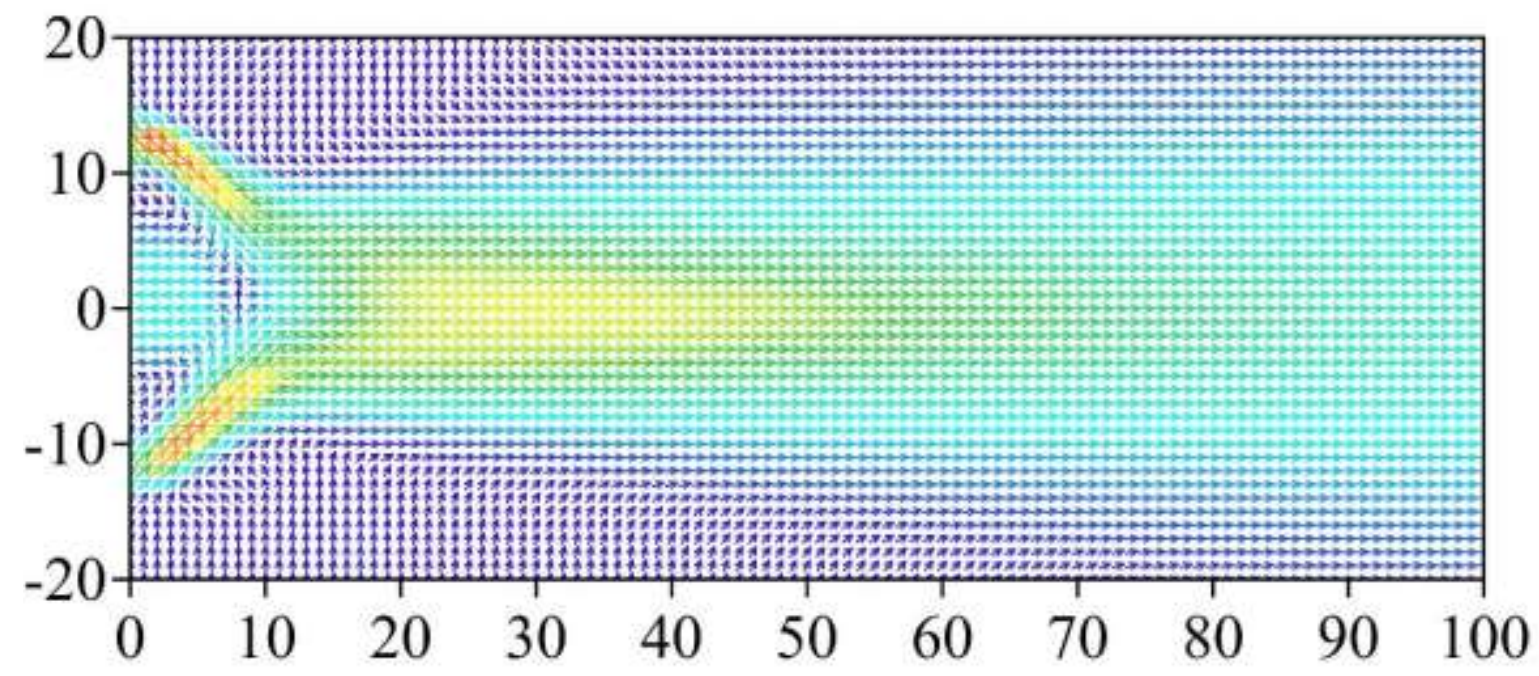

(a)

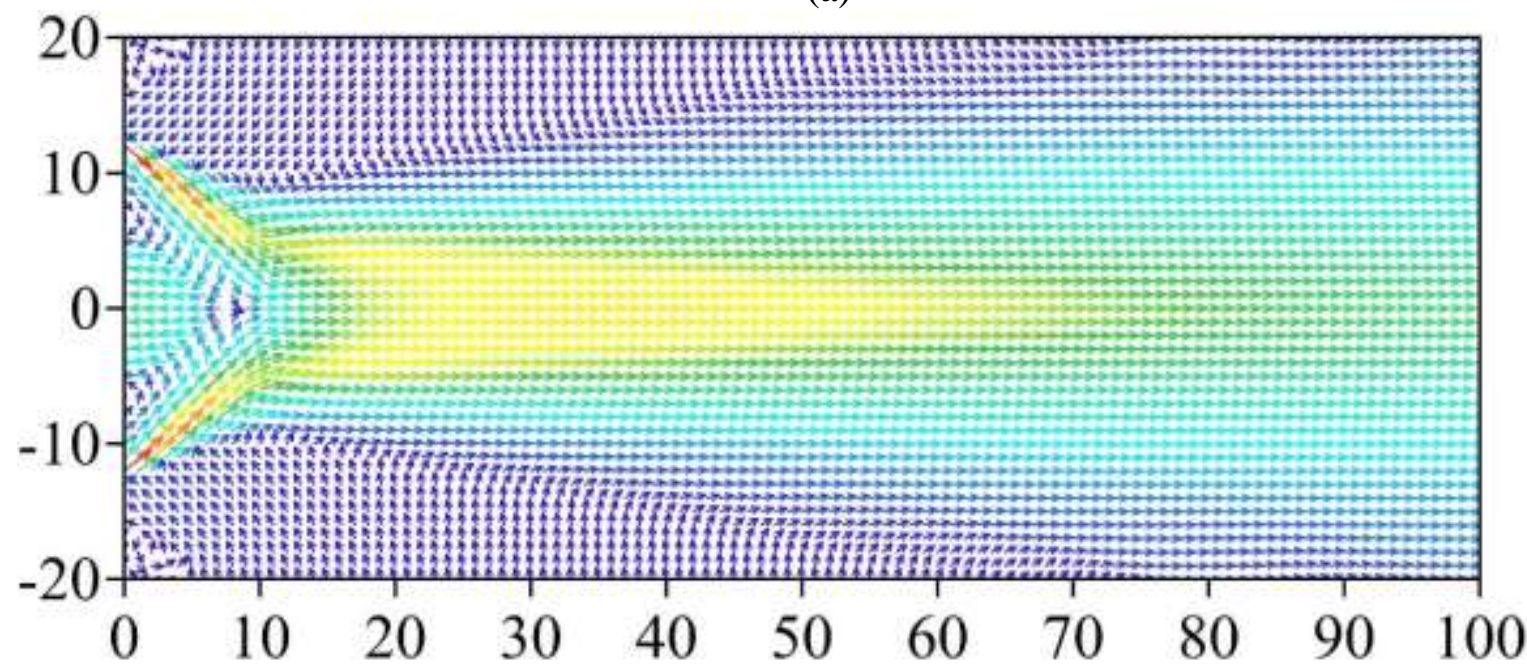

(b) 


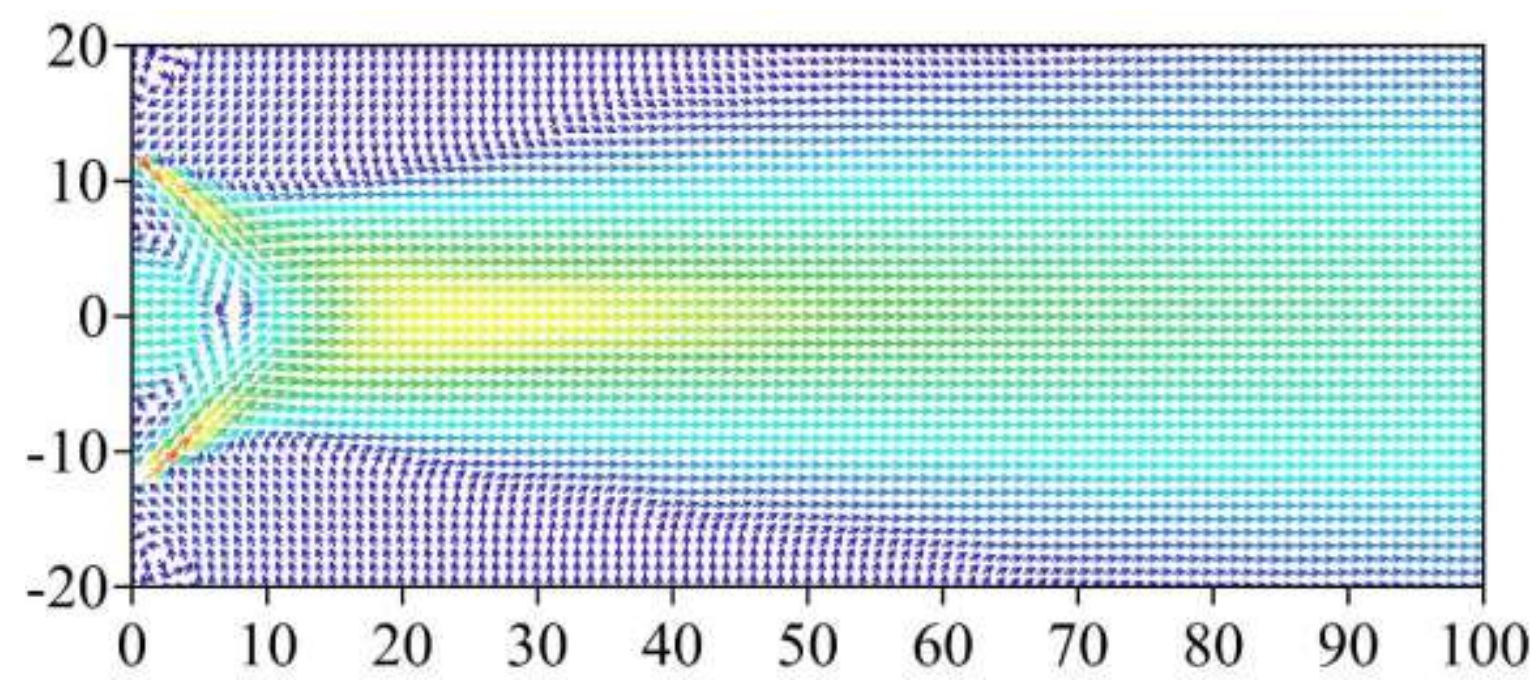

(c)

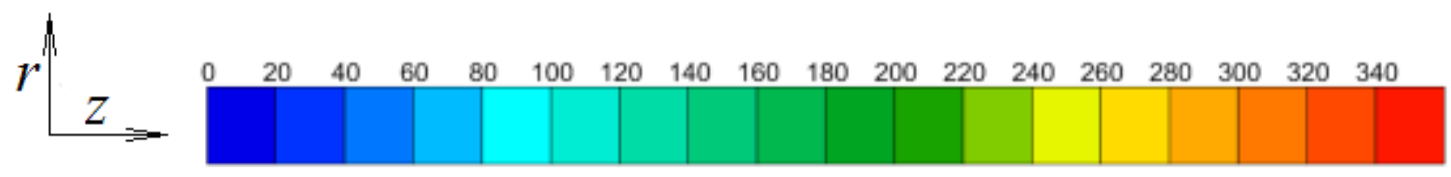

Fig. 7. Vector field of average velocity, $\mathrm{m} / \mathrm{s}\left(P_{0}=1 \mathrm{bar}\right)$ : (a) experiments; (b) calculation using the RSM model of turbulence; (c) calculation using the DES model of turbulence.

According to results of experiments and numerical simulation, the flow structure depends significantly on the air pressure in the nozzle. Velocity distributions at the excess pressure in the nozzle $P_{0}$ $=1$ bar are shown in Figs. 7 and 8. At this pressure, an acoustic gas jet is formed at the outlet of the annular nozzle, the Mach number on the nozzle edge is $M \sim 1.0$. In the zone of intersection (at $z=10 \mathrm{~mm}$ near the axis of symmetry), the converging annular air jet becomes concentrated and forms a direct axial jet and return (cumulative) jet. A straight axial jet expands; the flow in it becomes subsonic with the Mach number $\mathrm{M} \sim 0.6$.
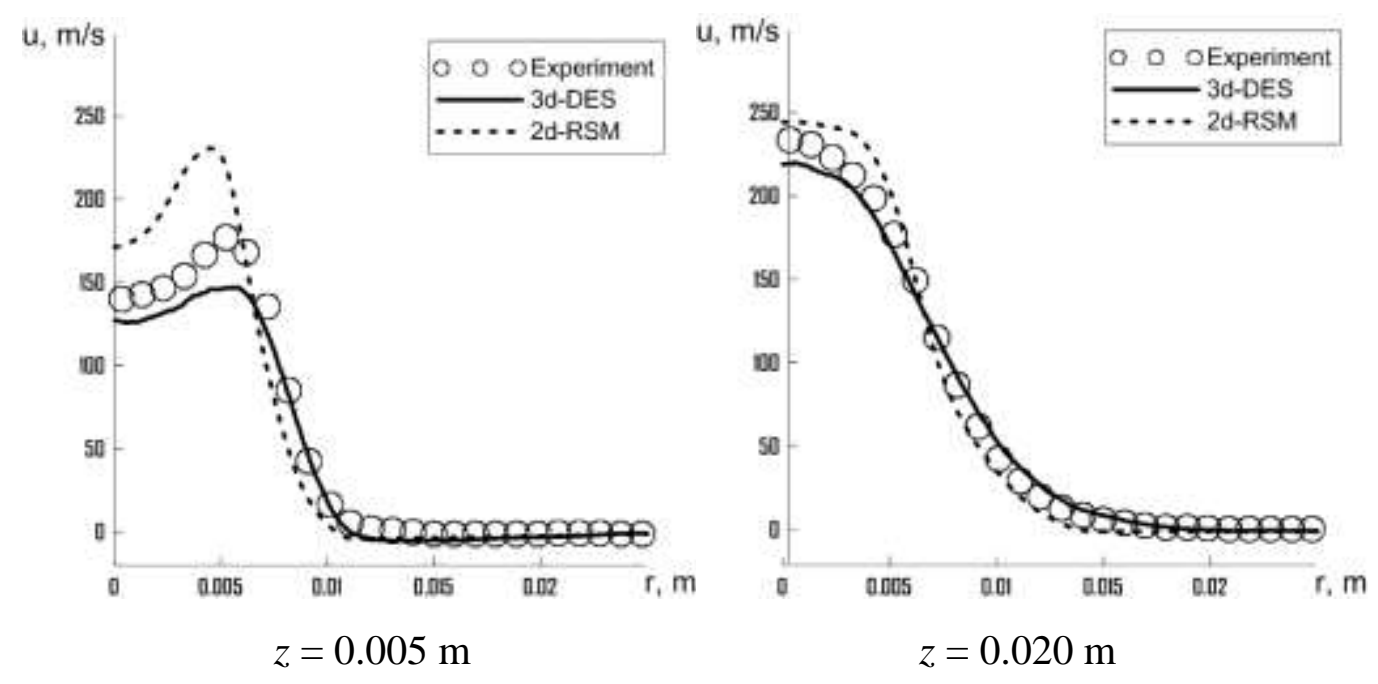

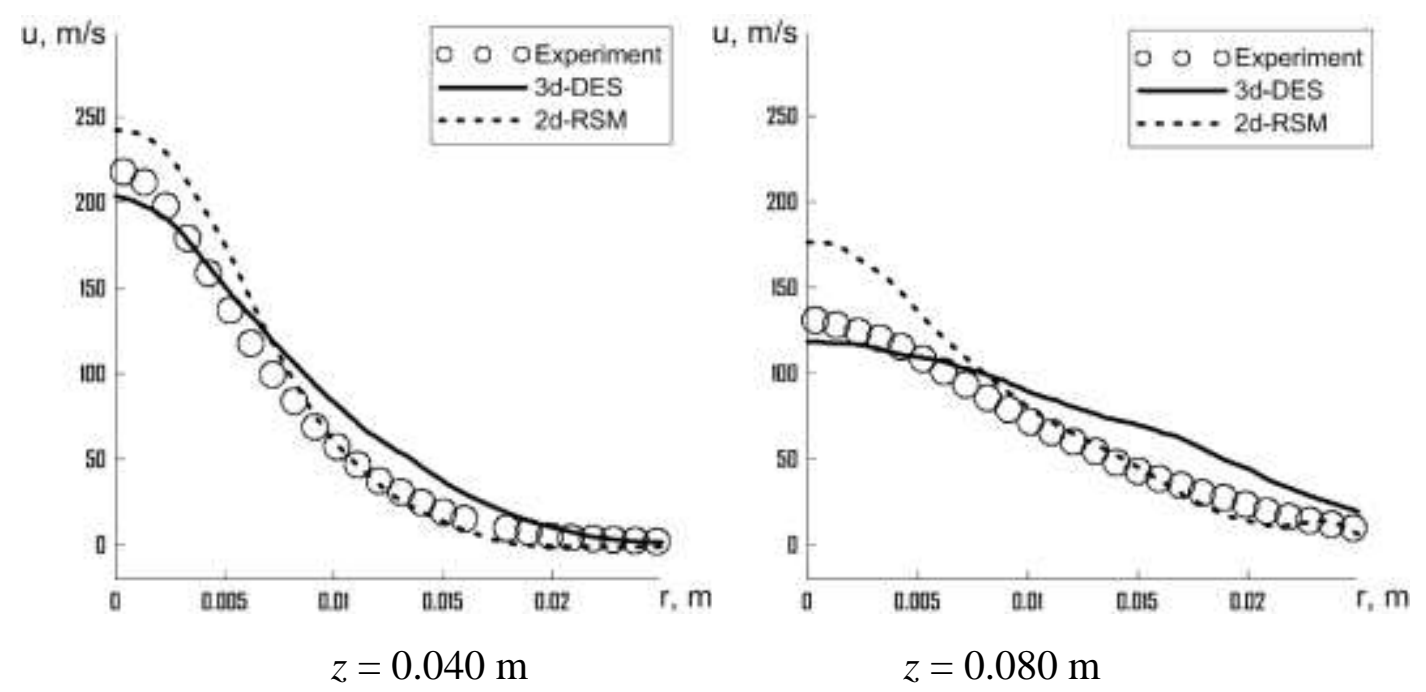

Fig. 8. Profiles of axial velocity $(u)$ at different distances from the nozzle $\left(P_{0}=1 \mathrm{bar}\right)$.

Comparison of calculation results with the experiment showed that calculation is qualitatively and quantitatively consistent with the PIV measurements. According to comparison of the axial velocity profiles (Fig. 8), in general, the DES model describes experimental data for the subsonic flows better than the RSM model. The RSM model overestimates velocity on the jet axis, but closer to the jet boundary, it provides better match with the experiment.

The streamlines and isosurface of the Q-criterion, which visualizes the large-scale vortex structures in the flow, are presented in Fig. 9. The Q-criterion reflects the balance between the rotation and strain rate intensity at a local flow point $\mathrm{Q} \equiv 1 / 2\left(\boldsymbol{\Omega}^{\mathbf{2}}-\boldsymbol{S}^{\mathbf{2}}\right)$, where $\boldsymbol{S}$ and $\boldsymbol{\Omega}$ are the symmetric and antisymmetric parts of the velocity gradient tensor $\nabla \mathbf{u}\left(S_{i, j}=1 / 2\left(u_{i, j}+u_{j, i}\right), \Omega_{i, j}=1 / 2\left(u_{i, j}-u_{j, i}\right)\right)$. According to [24], the regions of $\mathrm{Q}>0$ identify the presence of a vortex motion. According to the results, the flow has a specific structure inside the conical diffuser (see Fig. 9). Due to the action of the cumulative return jet, a toroidal vortex with a return flow on the axis is formed there. The obtained results confirm the authors' hypothesis about the flow structure described above and characterize the operating principle of the studied nozzle. In the case of liquid supply through a central channel, this arrangement of aerodynamics will ensure efficient spraying with formation of a finely dispersed gas-droplet flow [25-26]. 


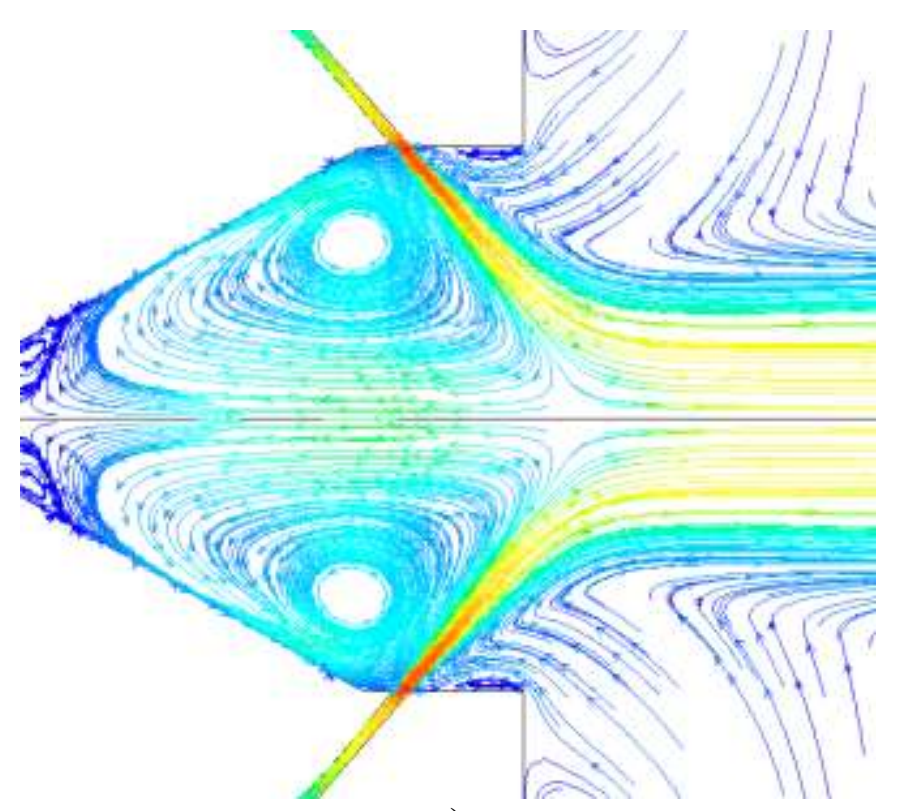

a)

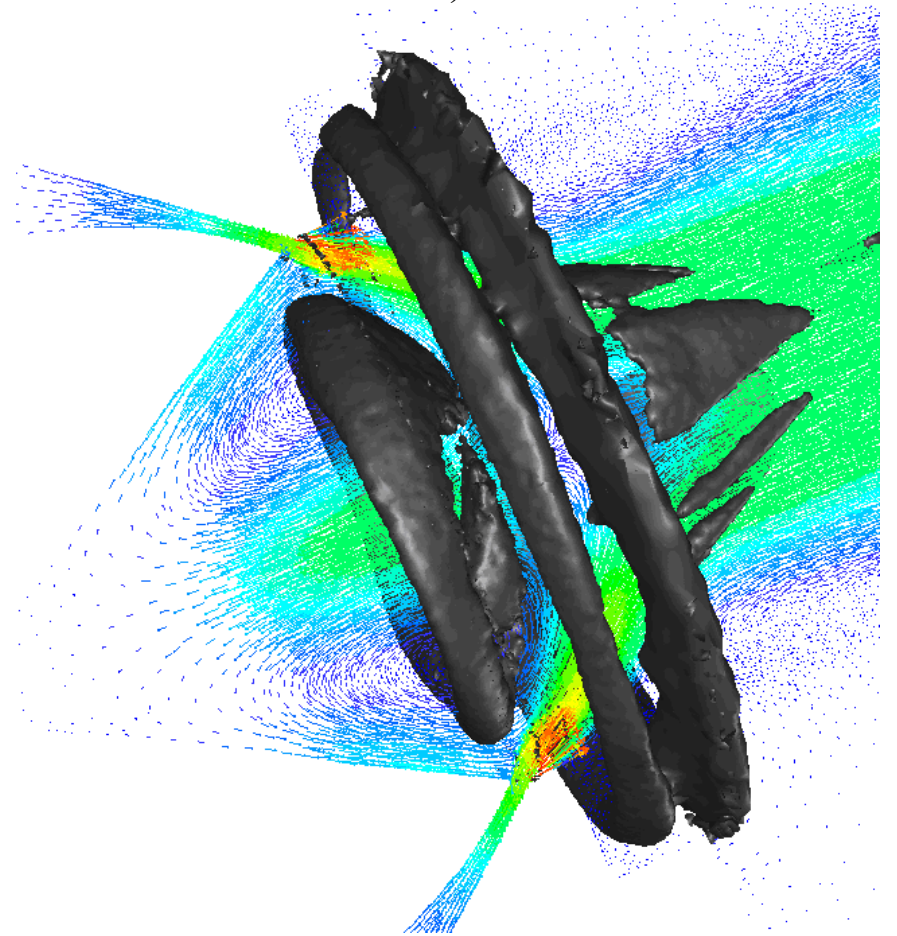

b)

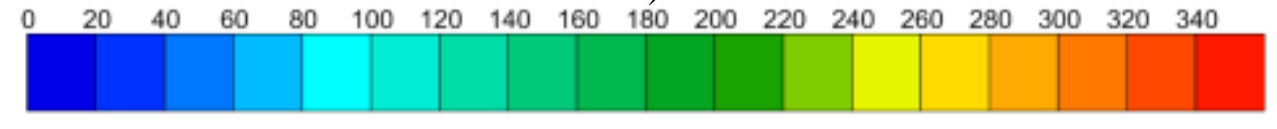

Fig. 9. Flow structure in the nozzle diffuser at $P_{0}=1$ bar:

(a) - streamlines, RSM model; (b) - vector field of velocity $(\mathrm{m} / \mathrm{s})$ and isosurface of normalized $Q$-criterion $(Q=0.3)$, DES model.

If the air pressure in the nozzle is $P_{0}=2$ bars (Figs. 10, 11), the flow structure, in general, is qualitatively similar to the flow at $P_{0}=1$ bar. However, the annular jet becomes supersonic, and the Mach number on the nozzle edge is $\mathrm{M}$ 1.4. The density contours (Fig. 10) show that the annular jet flowing out of the gas nozzle consists of alternating oblique shock waves. The presence of these shock waves increases 
the efficiency of liquid dispersion and droplet crushing. It should be noted that at $P_{0}=2$ bars, the axial jet is still subsonic, and the Mach number in this jet is $\mathrm{M} \sim 0.8$. (Fig. 11).

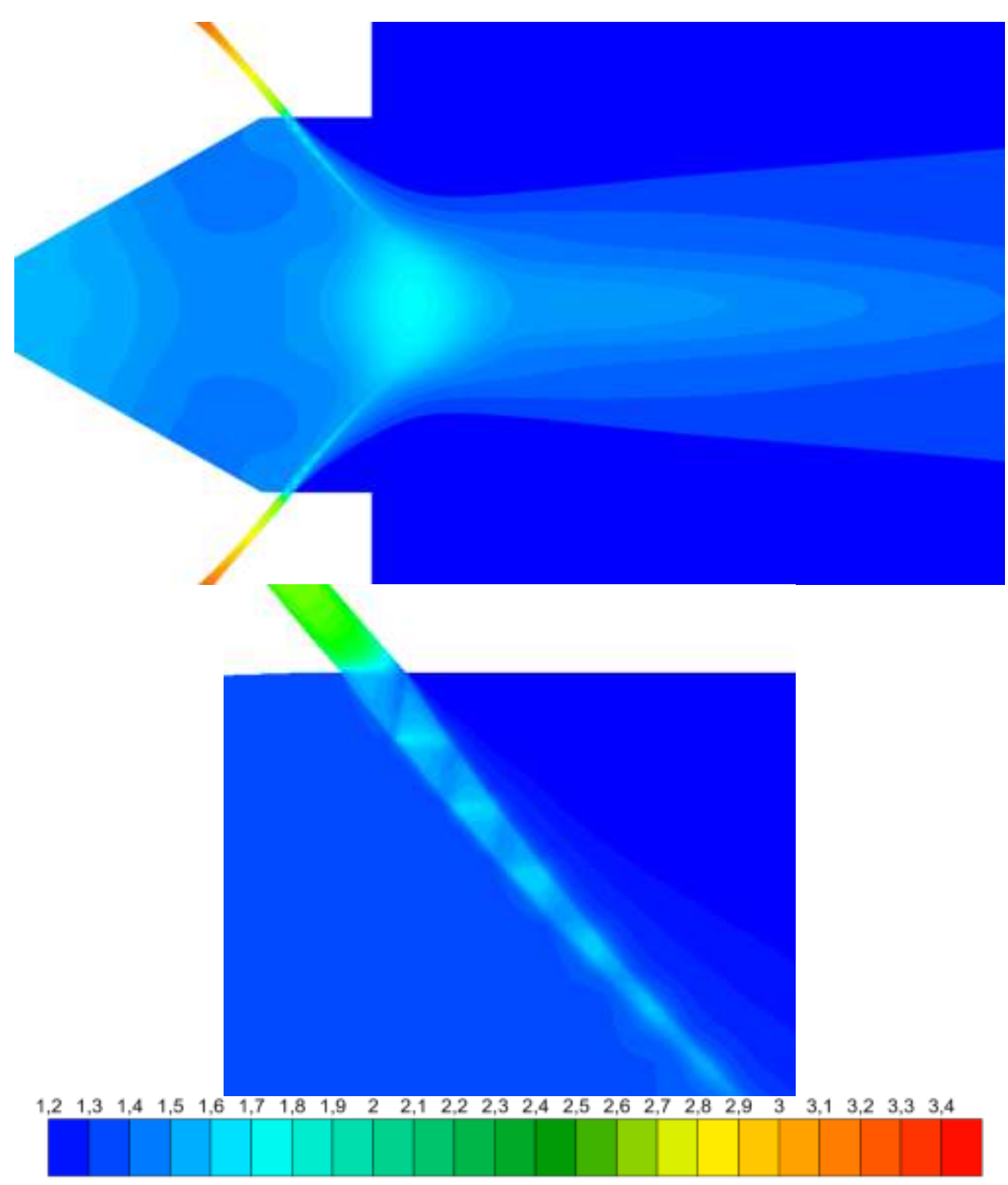

Fig. 10. Contours of density at $P_{0}=2 \mathrm{bar}, \mathrm{kg} / \mathrm{m}^{3}$ 


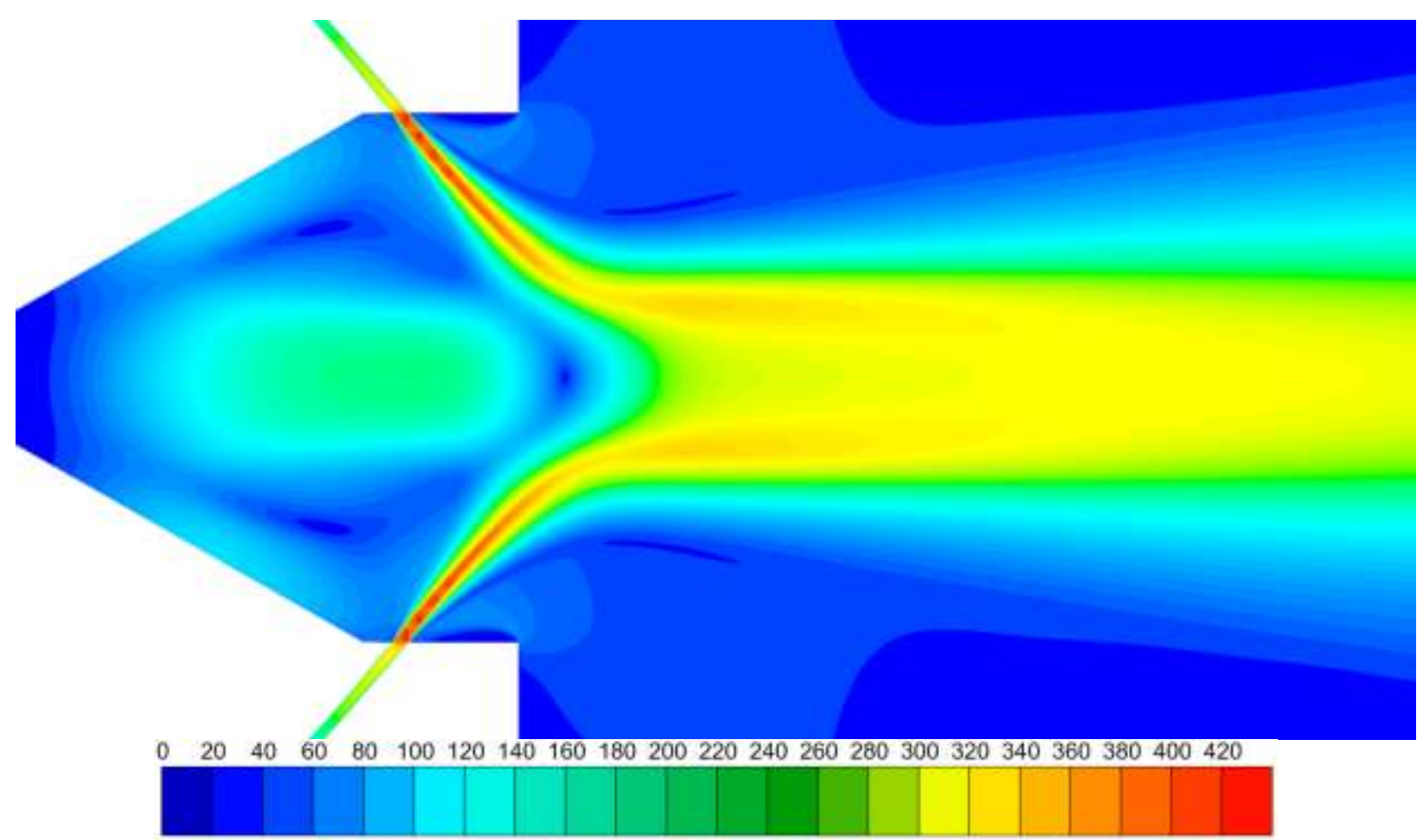

Fig. 11. Contours of velocity module at $P_{0}=2$ bar, $\mathrm{m} / \mathrm{s}$.

With an excess pressure increase in the nozzle to $P_{0}=3$ bars, the annular jet expands faster. More significant changes occur with the cumulative axial jet. Starting with excess pressure $P_{0}=3$ bars, the axial jet becomes supersonic (Figure 12), $\mathrm{M} \sim 1.3$, but despite this, there is no a pronounced shock-wave structure in it. Both experiments and calculations demonstrate this.

The results of a quantitative comparison of calculation and experiment are shown in Fig. 13. It can be seen that calculation describes the structure of the annular jet well. At that, in general, the results of calculations for the supersonic flow by the RSM model describe the PIV measurements better than the DES model. Calculation by the DES model gives more intensive braking of the axial jet. The difference is already observed at the very beginning of the axial jet, but not in the mixing layer. In the authors' opinion, the main reason relates to the fact that gradient adaptation of the calculated grid was not used for the DES calculations, in contrast to the two-dimensional RSM model. Therefore, the detailed elaboration of the threedimensional computational grid for the DES model was not sufficient for good resolution of the shock-wave cells in the annular jet, and this affected the quality of the axial jet calculation. 


\section{Experiment}

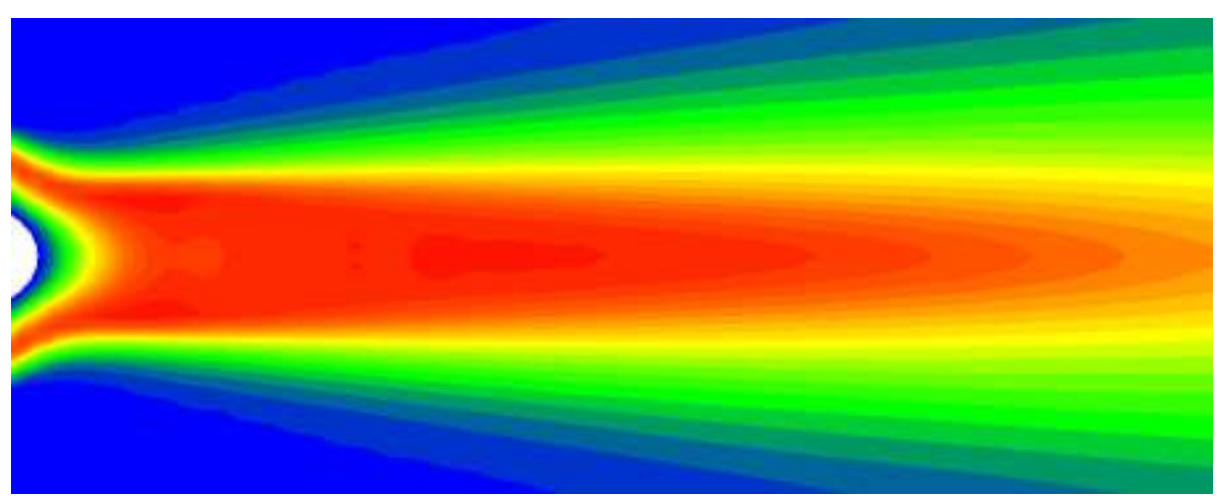

\section{RSM}

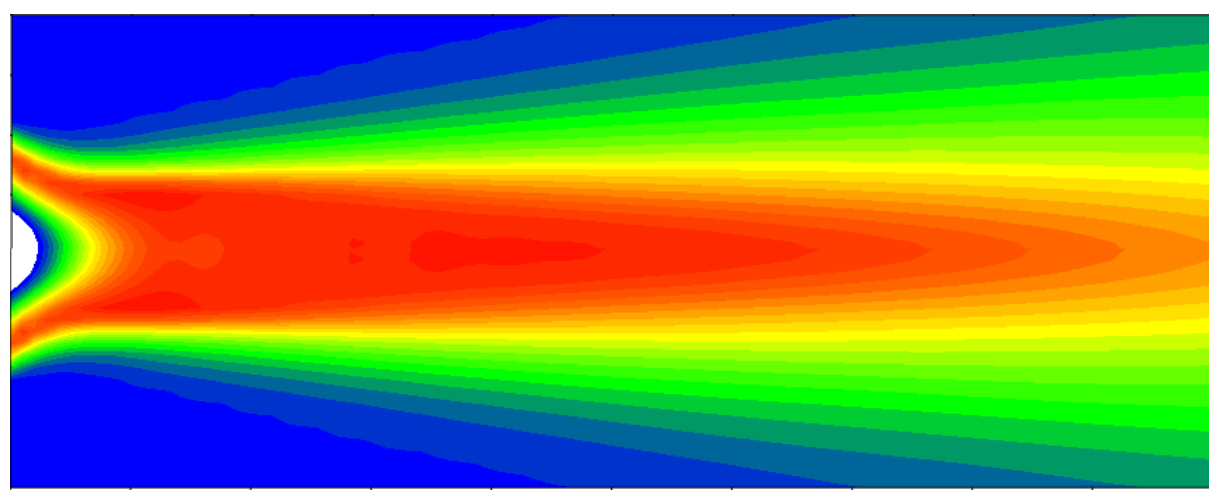

\section{DES}

$-30-1010 \quad 3050 \quad 70 \quad 90110130150170190210230250270290310330350370390410430450$

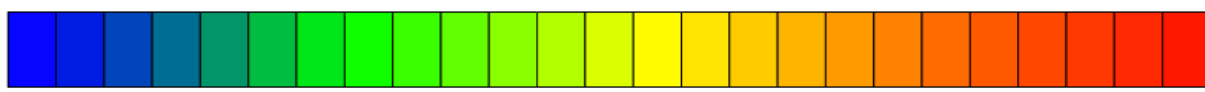

Fig. 12. Distribution of axial velocity component $(u), \mathrm{m} / \mathrm{s}\left(P_{0}=3 \mathrm{bar}\right)$. 

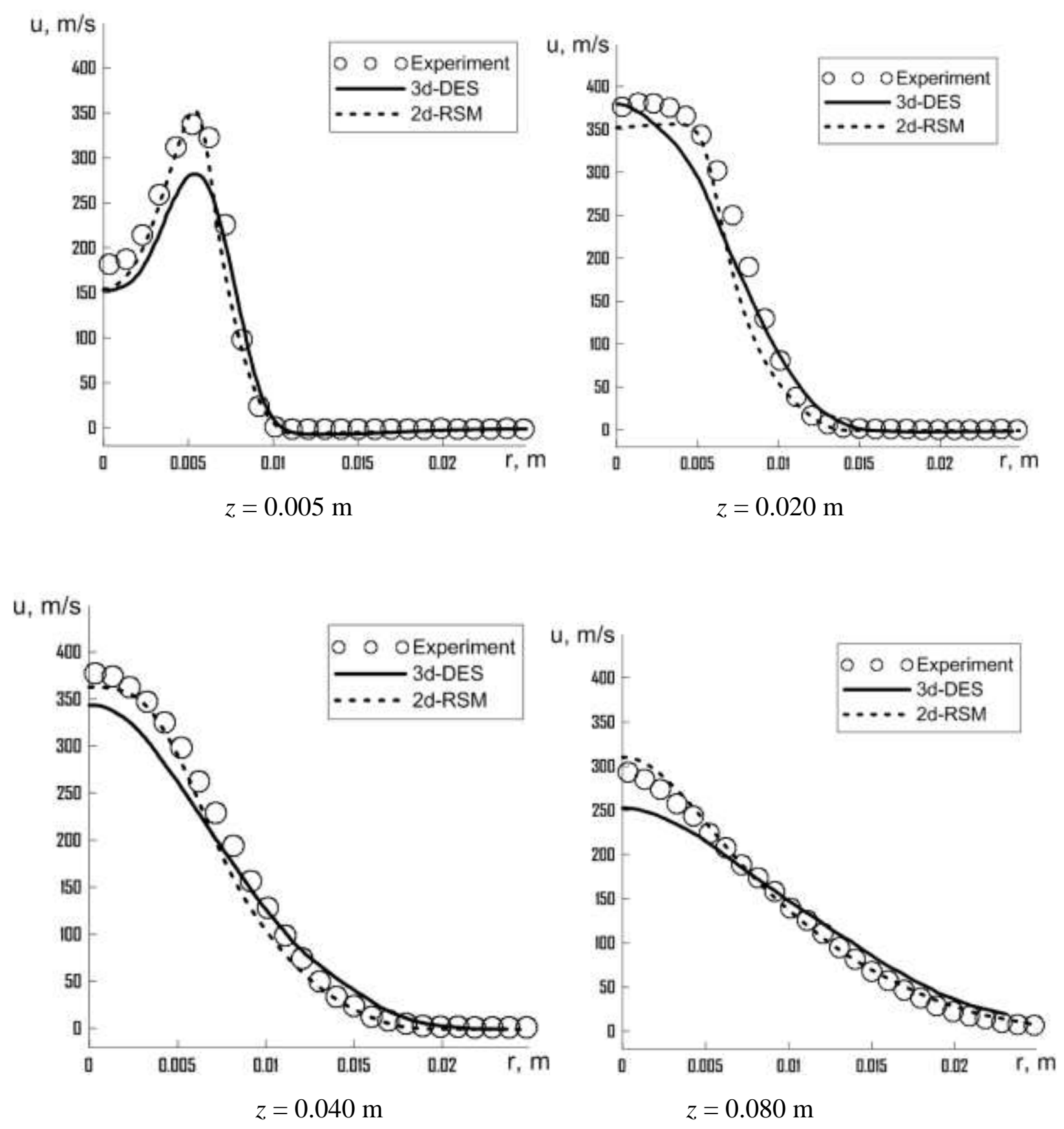

Fig. 13. Profiles of axial velocity $(u)$ at different distances from the nozzle $\left(P_{0}=3\right.$ bar).

The results of calculations performed for the regimes with a further increase in the air pressure in the nozzle $\left(P_{0}>3\right.$ bars $)$ showed that the flow pattern changes significantly. Distribution of gas dynamic parameters for the excess air pressure in the nozzle $P_{0}=5$ bars is shown in Figs. 14, 15. Due to impossibility to calculate the flow, starting from the outlet section of the annular nozzle, the velocity vector in the supersonic ring jet has a radial component directed away from the axis. As a result, at pressure $P_{0}>4$ bars, the boundary of the axial jet in the initial section takes a barrel-like shape. As it moves downstream, the radial component of the velocity vector changes periodically its direction, which contributes to formation of a multi-cellular shock-wave structure of an underexpanded jet. Near the jet boundary, the streamlines acquire a certain curvature, whose value depends on the pressure drop at the nozzle outlet and initial state of 
the boundary layer of the jet. The presence of shock-wave cells at the axial jet periphery (Fig. 15) will contribute to further additional fragmentation of liquid fuel droplets.

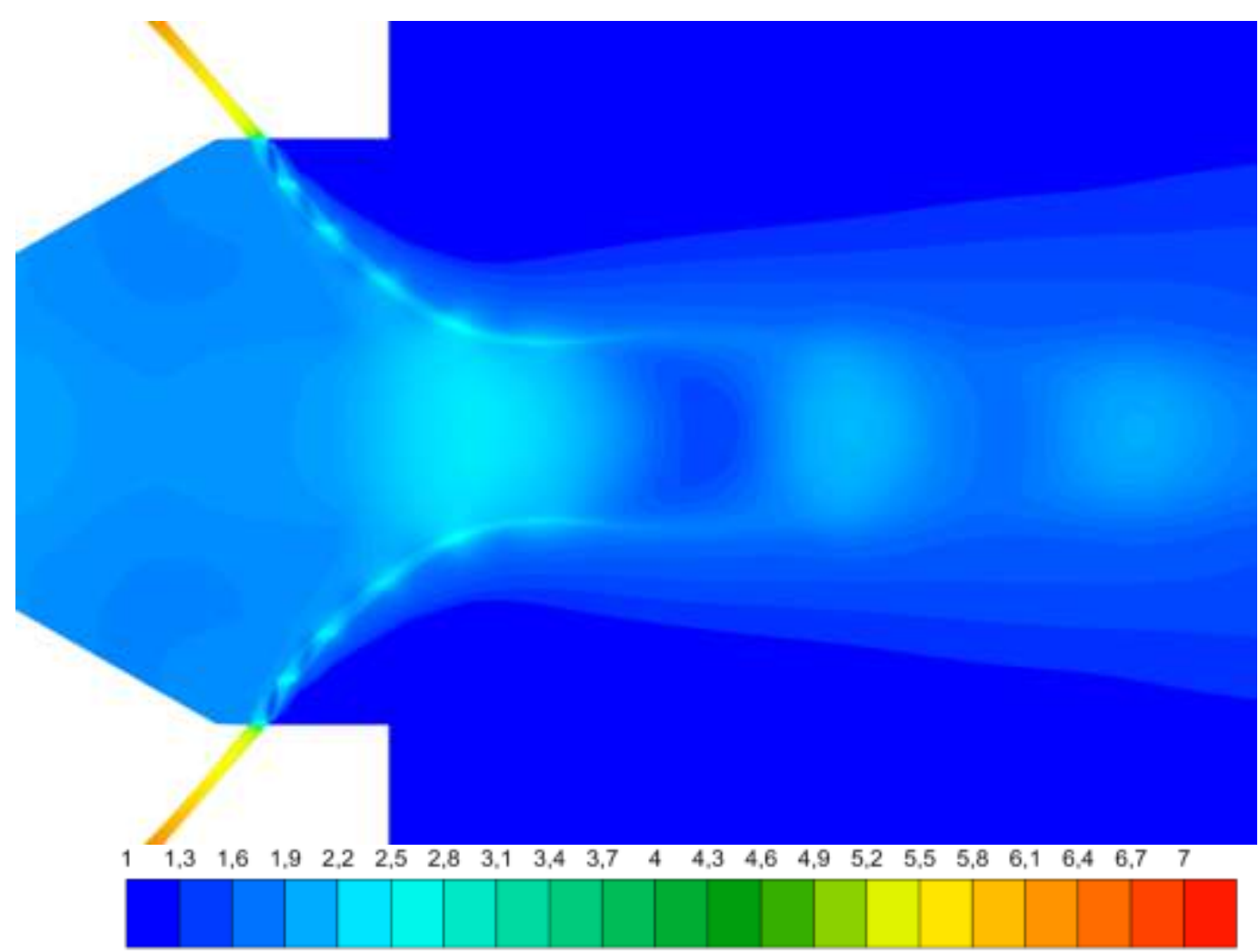

Fig. 14. Contours of density at $P_{0}=5 \mathrm{bar}, \mathrm{kg} / \mathrm{m}^{3}$. RSM model.

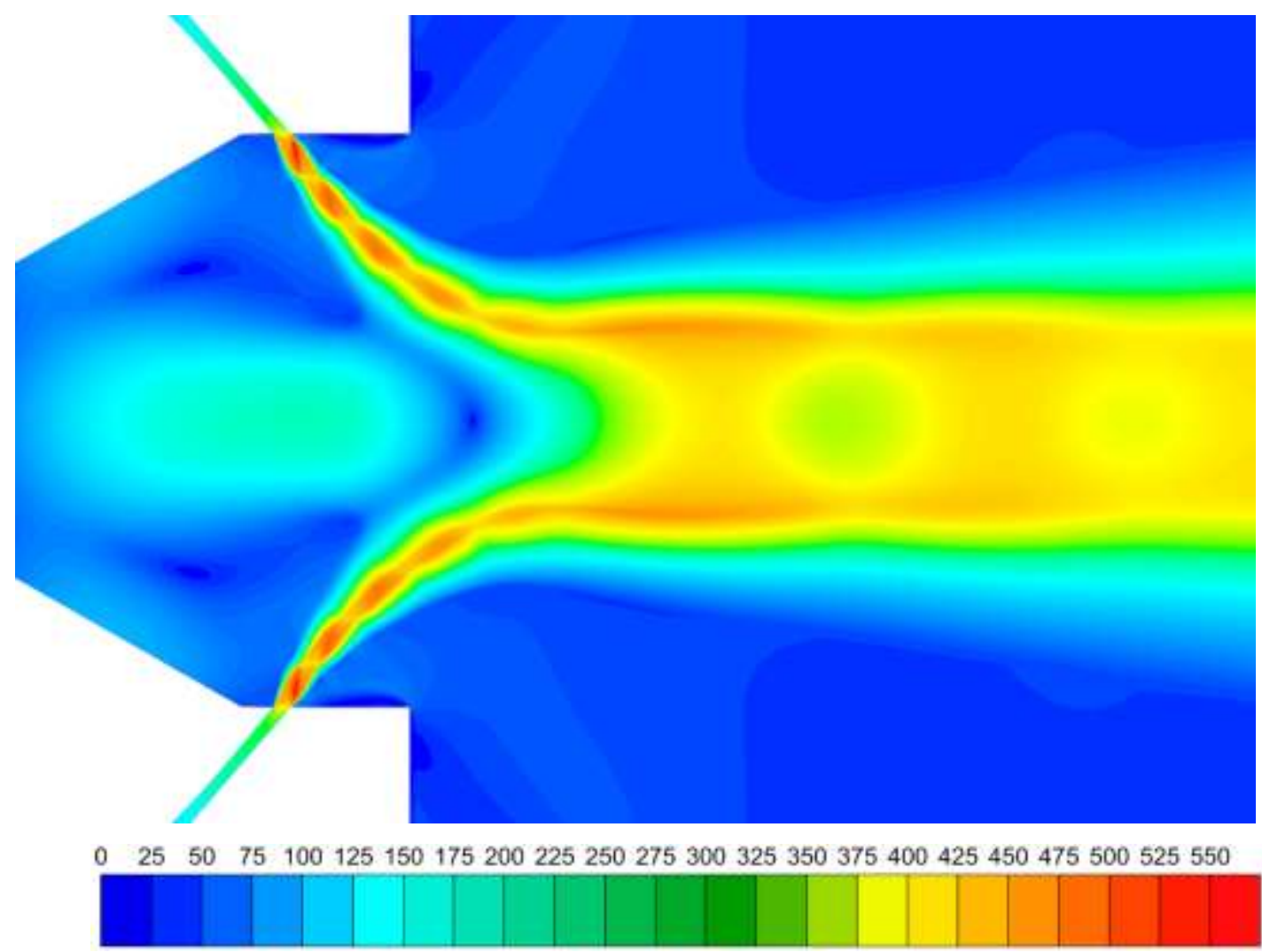

Fig. 15. Contours of velocity module, $\mathrm{m} / \mathrm{s}\left(P_{0}=5\right.$ bar $)$. RSM model. 
With an increase in pressure to $P_{0} \geq 55$ bars, the flow structure as a whole is similar to that described above. However, at the end of the first "barrel" of the axial jet, the Mach discs start forming for the first time (see Fig. 15). In the region of the first "barrel" of the jet, there is greatest maldistribution of the gas-dynamic parameters, both in longitudinal and transverse directions. At the large distances from the nozzle edge, the wave processes become weaker, the pressure in the jet is equalized, and the flow acquires an isobaric character. The annular jet at high pressures $\left(P_{0}>5\right.$ bars $)$ expands significantly. The barrel-like shape of the non-isobaric jet boundary causes the appearance of shock waves of a similar configuration, and reflection of the latter from the axis can lead to formation of configurations with direct closing shock waves.

The calculated velocity of the working gas outflow from the annular nozzle under the studied regimes (for the pressure in the nozzle $P_{0}=0.5-5.0$ bars) was $260-550 \mathrm{~m} / \mathrm{s}$.

\section{Conclusion}

In this paper, the structure of a single-phase gas flow from a pneumatic nozzle for liquid fuel spraying was studied using the PIV measurements and numerical simulation. To model turbulence, the Reynolds stress model (RSM) in stationary axisymmetric formulation and the DES method were used. The gasdynamic characteristics of the flow were studied for various regimes with an excess air pressure in the nozzle from 1 to 5 bars. The results of numerical simulation are compared with the PIV measurements. Good qualitative and quantitative agreement between calculation and experiment was obtained, both for subsonic and supersonic outflows.

It is shown that in the entire investigated range of excess air pressure in the nozzle near the axis of symmetry, the converging annular jet becomes concentrated and forms the straight and recurrent cumulative axial jets. Due to interaction of the return and annular jets, a toroidal vortex is formed in the diffuser. At operation of the liquid fuel nozzle, such a return flow will contribute to the effective destruction of the liquid jet and formation of a finely dispersed two-phase flow. Some drops enter the recirculation zone, where they are ejected by an annular jet with further crushing.

It has been determined that even at excess air pressure in the nozzle $P_{0}=1 \mathrm{bar}$, an acoustic annular converging jet is formed at the nozzle outlet; the direct axial jet expands and its flow becomes subsonic, with the Mach number $\mathrm{M} \sim 0.6$. With a further increase in $P_{0}$, a supersonic jet flowing out of the annular nozzle into a flooded space is characterized by significant maldistribution of gas-dynamic quantities, both along the axis and in transverse direction. In this case, the annular jet contains alternating oblique shock waves. The flow in a direct axial jet is also characterized by the presence of shock-wave structures. With an increase in pressure $P_{0} \geq 4$ bars, the boundary of the axial jet in the initial section takes a barrel-like shape, and the Mach discs start forming. The complex spatial structure of the flow (both in the toroidal vortex and outside it) ensures efficient dispersion of liquid fuel in this nozzle. 
The obtained results quite fully characterize aerodynamics of the single-phase flow created by the studied pneumatic nozzle and allow predicting the regimes for studying the gas-droplet flows in order to optimize nozzle operation, which is the goal of the next stage of research.

The work was supported by the Russian Foundation for Basic Research (project No. 18-3800153-mol_a). 


\section{REFERENCES}

1. Maltsev L.I., Kravchenko I.V., Lazarev S.I., Lapin D.A. Combustion of black coal in the form of coal-water slurry in low capacity boilers // Thermal Engineering. 2014. Vol.61. No.7. P.486-490.

2. Khodakov G.S. Coal-water suspensions in power engineering // Thermal Engineering. 2007. Vol.54. No.1. P.36-47.

3. Trubetskoi K.N., Zaidenvarg V.E., Kondrat'ev A.S., Murko V.I., Kassikhin G.A., Nekhoroshii I.Kh. Water-coal fuel: the results of technology development and perspectives of its utilization in Russia // Thermal Engineering. 2008. Vol.55. No.5. P.413-417.

4. Glushkov D.O., Shabardin D.P., Strizhak P.A., Vershinina K.Y. Influence of organic coal-water fuel composition on the characteristics of sustainable droplet ignition // Fuel Processing Technology. 2016. Vol.143. P.60-68.

5. Glushkov D.O., Strizhak P.A., Chernetskii M.Yu. Organic coal-water fuel: Problems and advances (Review) // Thermal Engineering. 2016. Vol.63. No.10. P.707-717.

6. Aiuchi K., Moriyama R., Takeda S., Kitada S., Onozaki M., Katayama Y. A pre-heating vaporization technology of coal-water slurry for thegasification process // Fuel Processing Technology. 2007. Vol. 88. No.4. P.325-331.

7. Wang R., Liu J., Gao F., Zhou J., Cen K. The slurrying properties of slurry fuels made of petroleum coke and petrochemical sludge // Fuel Processing Technology. 2012. Vol.104. P.57-66.

8. Chen X., Zhao L., Zhang X., Qian C. An investigation on characteristics of coal-water slurry prepared from the solid residue of plasma pyrolysis of coal // Energy Conversion and Management. 2012. Vol. 62. P.70-75.

9. Tu Y., Xu Z., Wang W. Method for evaluating packing condition of particles in coal water slurry // Powder Technology. 2015. Vol.281. P.121-128.

10. He Q., Xie D., Xu R., Wang T., Hu B. The utilization of sewage sludge by blending with coal water slurry // Fuel. 2015. Vol.159. P.40-44.

11. Russian Federation Patent No.2346756 (2007), Pneumatic injector.

12. Alekseenko S., Kravchenko I., Maltsev L. Combustion technology for water-fuel mixtures // Proceedings of the 2012 International Conference on Thermal Treatment Technologies \& Hazardous Waste Combustors, October 22-24, 2012, New Orleans, LA. Paper No. 25. - 19 p.

13. Alekseenko S.V., Anufriev I.S., Vigriyanov M.S., Dulin V.M., Kopyev E.P., Sharypov O.V. Steam enhanced regime for liquid hydrocarbons combustion: velocity distribution in the burner flame // Thermophysics and Aeromechanics. 2014. Vol.21. No.3. P.393-396.

14. Scarano F. Overview of PIV in supersonic flows // Topics in applied physics. 2008. Vol.112. P.445463.

15. Gibson M.M., Launder B.E. Ground Effects on Pressure Fluctuations in the Atmospheric Boundary Layer // J. Fluid Mech. 1978. Vol.86. P.491-511. 
16. B. E. Launder, G. J. Reece, and W. Rodi. "Progress in the Development of a Reynolds-Stress Turbulence Closure". J. Fluid Mech. 68(3). 537-566. April 1975.

17. B.J. Daly and F.H. Harlow. Transport Equations in Turbulence. The Physics of Fluids, 13(11), 1970.

18. Speziale C.G., Sarker S., Gatski T.B. Modelling the pressure-strain correlation of turbulence. An invariant dynamical systems approach // J. Fluid Mech. 1991. Vol.227. P.245-272.

19. Strelets M. Detached Eddy Simulation of Massively Separated Flows // AIIA Paper. 2001 №2001 0879. $-19 \mathrm{p}$.

20. Menter F.R. Two Equation Eddy Viscosity Turbulence Models for Engineering Applications // AIAA J. 1994. Vol.32. No.8. P.1598-1605.

21. Minakov, A.V., Sentyabov, A.V., Platonov, D.V., Dekterev, A.A., Gavrilov, A.A. Numerical modeling of flow in the Francis-99 turbine with Reynolds stress model and detached eddy simulation method // Journal of Physics: Conference Series. 2015. Vol.579, Issue 1, 012004.

22. Minakov, A.V., Platonov, D.V., Dekterev, A.A., Sentyabov, A.V., Zakharov, A.V. The numerical simulation of low frequency pressure pulsations in the high-head Francis turbine // Computers and Fluids. 2015. Vol.111. P.197-205.

23. Minakov, A.V., Platonov, D.V., Litvinov, I.V., Shtork, S.I., Hanjalić, K. Vortex ropes in draft tube of a laboratory Kaplan hydroturbine at low load: an experimental and LES scrutiny of RANS and DES computational models // Journal of Hydraulic Research. 2017. Vol.55. Issue 5. P.668-685.

24. Hunt J.C.R., Wray A.A. and Moin P. Eddies, streams, and convergence zones in turbulent flows // Center For Turbulence Research. Proceedings of the Summer Program 1988. pp. 193-208.

25. Alekseenko S., Maltsev L., Bogomolov A., Chernetskiy M., Kravchenko I., Kravchenko F., Lapin D., Shevyrev S., Lyrschikov S. Results of pilot-operating combustion of coal-water fuel in a lowcapacity hot water boiler // Bulletin of the Tomsk Polytechnic University. Geo Assets Engineering. 2017. Vol.328. Issue 12. P.16-28.

26. Glushkov D., Kuznetsov G., Chebochakova D., Lyakhovskaya O., Shlegel N., Anufriev I., Shadrin E. Experimental study of coal dust ignition characteristics at oil-free start-up of coal-fired boilers. Applied Thermal Engineering. Volume 142, September 2018, Pages 371-379 